\title{
LYAPUNOV-SCHMIDT METHOD DEDICATED TO THE OBSERVER ANALYSIS AND DESIGN
}

\author{
D. BENMERZOUK AND J. P. BARBOT
}

Received 14 June 2005; Revised 14 February 2006; Accepted 5 March 2006

An analysis of observation error dynamics and an observer design are presented using the so-called Lyapunov-Schmidt method, firstly in order to analyze the solution's bifurcation of the observation error dynamics, and secondly in order to propose a robust observer with respect to such solution's bifurcation. Two numerical examples based on the Galerkin method show the pertinence of the proposed approach.

Copyright (c) 2006 D. Benmerzouk and J. P. Barbot. This is an open access article distributed under the Creative Commons Attribution License, which permits unrestricted use, distribution, and reproduction in any medium, provided the original work is properly cited.

\section{Introduction}

In this paper, the (so-called) Lyapunov-Schmidt method is used in order to analyze the solution's bifurcation of the observation error dynamics [3]. This method relies on Fredholm operators of finite index defined in Banach spaces which are immerged in appropriate Hilbert spaces in order to use two projections allowing to transform an infinite dimensional problem into a finite one. Following this, a bifurcation equation is established, solving this equation gives us the number of solutions of the problem (i.e., the number of solutions of the observation error dynamics). Obviously, a solution bifurcation is less known than the stability $[1,2,5,12]$, controllability $[8,6,7]$ and observability [4] bifurcations. Nevertheless, it is of prior importance to know if the real solution is or is not in the neighborhood of the solution obtained with a linear approximation. Roughly speaking, we call solution bifurcation the nonexistence or the existence of two solutions in the vicinity of the linear one. This phenomena may appear as a purely mathematical interest, but obviously, it is also often an important problem in control systems theory. For example, it is usual to design an observer for nonlinear system on the basis of a linear approximation. It is then expected that the error dynamics have a solution closed to the linear one. This is not obvious, especially when considering the fact that the initial value of the nonlinear system is totally or partially unknown. In this paper, an analysis of the observer design is proposed on the basis of the Lyapunov-Schmidt approach.

Hindawi Publishing Corporation

Mathematical Problems in Engineering

Volume 2006, Article ID 43681, Pages 1-28

DOI 10.1155/MPE/2006/43681 
Moreover, an academic example of observability analysis in the case of unidimensional nonlinear systems is given. After that, we highlight the technological interest of the proposed approach by considering an analysis and an observer design for a two dimensional Lagrangian system.

The paper is organized as follows: in Section 2, a solution's bifurcation is studied using Lyapunov-Schmidt method. An observer analysis and the related design are introduced in Section 3. In Section 4, two numerical examples using the Galerkin method [11] are developed in order to highlight the efficiency of the proposed analysis and the interest of the approach.

\section{Problem analysis using Lyapunov-Schmidt method}

Lyapounov-Schmidt method [10] essentially allows to carry out a local study of nonlinear system's solution in the neighborhood of an equilibrium point near the linear one. It is important to note that a function solving the problem and depending on perturbation parameters is determined on an open set of initial conditions.

2.1. Problem statement. Let us consider the following system:

$$
\dot{e}=A(t) e+\gamma(e, \chi, t)
$$

where $t \in J=[0, T], e: J \rightarrow M \subset \mathbb{R}^{n}$ is the state and $M$ is a nonempty open set of $\mathbb{R}^{n}$. $\chi: J \rightarrow \Omega \subset \mathbb{R}^{p}$ is an external signal and $\Omega$ is a nonempty open set of $\mathbb{R}^{p} . A: J \longrightarrow \mathbb{R}^{n \times n}$ is a continuous application. $\gamma: M \times \Omega \times J \rightarrow \mathbb{R}^{n}$ is an application regrouping all the nonlinearities and satisfying the following assumptions.

Assumption 2.1. $\gamma$ is $C^{2}$ with respect to $e$.

Assumption 2.2. $\int_{0}^{T} \gamma(0,0, t) d t=\int_{0}^{T}(\partial \gamma / \partial e)(0,0, t) d t=0$.

$e(0)$ is assumed to be free (i.e., not fixed) in the neighborhood of $e=0$.

Hereafter our purpose is to analyze the number of solutions for the system (2.1), in the neighborhood of a nontrivial solution $e_{L}$ of the related linear system defined as

$$
\dot{e}=A(t) e .
$$

2.2. Abstract problem formulation. Let $C_{n}^{0}:=C^{0}\left(J, \mathbb{R}^{n}\right)$ be the set of continuous functions defined on the time interval $J$ and having values in $\mathbb{R}^{n}$. Moreover, $C_{n}^{0}$ is provided with the uniform convergence norm given by

$$
e \in C_{n}^{0}:\|e\|_{\infty}:=\sup _{t \in I}\|e(t)\|_{0}
$$

where $\|\cdot\|_{0}$ is a norm defined on $\mathbb{R}^{n}$ and so $\left(C_{n}^{0},\|\cdot\|_{\infty}\right)$ is a Banach space [2]. 
Similarly, let $C_{n}^{1}=C^{1}\left(J, \mathbb{R}^{n}\right)$ be the set of $C^{1}$ functions defined on the time interval $J$ and having values in $\mathbb{R}^{n}$, the norm for $C_{n}^{1}$ is defined as follows:

$$
e \in C_{n}^{1}:\|e\|=\|e\|_{\infty}+\|\dot{e}\|_{\infty}
$$

and thus $\left(C_{n}^{1},\|\cdot\|\right)$ is also a Banach space [2].

$X \subset C^{0}(J, \Omega)$ denotes the set of continuous external signal $\chi$ defined on the time interval $J$ and having values in $\Omega$.

In our application to observer analysis, $\chi$ is the output signal. ( $\chi$ may be a known or unknown input perturbations, this in function of the considered problem.)

In order to compare the linear solution of (2.2) with the nonlinear one of (2.1), it is natural to recall the following results.

Proposition 2.3 [9]. A general solution of the linear equation (2.2) is given by

$$
e_{L}(t)=\phi(t) e_{L}(0), \quad t \in J
$$

where $\phi(t)$ is the fundamental matrix associated to the linear system (2.2).

Proposition 2.4 [9]. A function $e \in C_{n}^{1}$ is a solution of the nonlinear problem (2.1) if and only if it is a solution of the following equation:

$$
e(t)=e_{L}(t)+\int_{0}^{t} \phi(t) \phi^{-1}(s) \gamma(e(s), \chi(s), s) d s
$$

Proof. The function $e_{L}$ given by (2.5) being a solution of the linear problem (2.2), thus any solution of the nonlinear problem (2.1) may be written in the form of (2.6) [9].

Remark 2.5. The initial condition of the nonlinear system (2.1) (or (2.6)) noted by $e(0)$ is the same as the one of the linear system (2.2) (or (2.5)) noted by $e_{L}(0)$, nevertheless, in order to avoid any confusion both notations are kept.

The linear operator $\mathfrak{E}$ is now defined as follows:

$$
\begin{gathered}
\mathfrak{L}: C_{n}^{1} \longrightarrow C_{n}^{0}, \\
(\mathfrak{E} e)(t)=\dot{e}(t)-A(t) e(t),
\end{gathered}
$$

and $N G$ denotes the Nemystskii nonlinear operator [10] related to $\gamma$ :

$$
N G: C_{n}^{1} \times X \longrightarrow C_{n}^{0} \quad \text { such that } N G(e, \chi)(t)=\gamma(e(t), \chi(t), t) .
$$

Using these notations, we obtain the following proposition.

Proposition 2.6. Equation (2.1) is equivalent (equivalent means that each solution of (2.9) is also solution of (2.1) and reciprocally) to

$$
\mathfrak{E} e=N G(e, \chi), \quad e \in C_{n}^{1}, \chi \in X .
$$

The proof is directly deduced from (2.7) and (2.8). 
2.3. Bifurcation Analysis. When $e(0)=0$, the linear system (2.2) admits the null function as a trivial solution and consequently the Cauchy problem corresponding to the nonlinear system (2.1) (or equivalently (2.9)) has one and only one solution in the neighborhood of $e=0$. It is well known that for fixed $e_{0} \neq 0$, we obtain one and only one solution of (2.9) by elementary translation in the vicinity of 0 . Nevertheless, if $e(0)$ is free, the existence and uniqueness of the solution are not guaranteed: this case is the most interesting one, because it is due to the fact that the Kernel of the operator $\mathfrak{E}$ is of dimension $n$ and some solution's bifurcations may appear.

In order to apply the Lyapounov-Schmidt method, we must verify that $\mathfrak{E}$ has relevant features.

Proposition 2.7. (i) $\mathfrak{E}$ is a linear continuous and bounded operator.

(ii) $\operatorname{dim} \operatorname{Ker} \mathfrak{E}=\operatorname{codim} \operatorname{Im} \mathfrak{E}=n$ and $\mathfrak{E}$ is a Fredholm operator of index 0 .

(iii) There exists $\hat{H}_{1}: C_{n}^{1}=\operatorname{Ker} \mathfrak{E} \oplus \hat{H}_{1}$ and there exists $\hat{H}_{2}: C_{n}^{0}=\operatorname{Im} \mathfrak{E} \oplus \hat{H}_{2}$ such that

$$
\begin{gathered}
\forall e \in C_{n}^{1}, \quad \exists \alpha_{i} \in \mathbb{R}, i=1, \ldots, n, \quad \exists v \in \hat{H}_{1}: e=\sum_{i=1}^{n} \alpha_{i} \varphi_{i}+v, \\
\forall h \in C_{n}^{0}, \quad \exists h_{1} \in \operatorname{Im} \mathcal{E}, \quad \exists h_{2} \in \hat{H}_{2}: h=h_{1}+h_{2},
\end{gathered}
$$

where $\varphi_{i}, i=1, \ldots, n$, are the $n$-column vectors of the fundamental matrix $\varphi$.

Proof. (i) Results from the definition of $\mathfrak{E}$.

(ii) Ker $\mathfrak{E}$ is spanned by $\varphi_{i}(t), i=1, \ldots, n$, thus $\operatorname{dim} \operatorname{Ker} \mathfrak{E}=n$ and from the Fredholm alternative [10], problem $\mathfrak{E} e=N G(e, \chi)$ admits a solution if and only if

$$
\int_{0}^{T}\left\langle N G(e, \chi)(t), \varphi_{i}(t)\right\rangle d t=0, \quad i=1, \ldots, n
$$

where $\langle\cdot, \cdot \cdot\rangle$ is the inner product in $\mathbb{R}^{n}$.

So $\operatorname{dim} \operatorname{Ker} \mathfrak{E}=$ codim $\operatorname{Im} \mathfrak{E}=n$ and Index $\mathfrak{E}=0$.

(iii) As Ker $\mathfrak{E}$ and Im $\mathfrak{E}$ have finite dimension and finite codimension, respectively, and since they are closed, it is possible to define continuous projections over each one. Let us consider the application $P_{0}$ given by

$$
\begin{gathered}
P_{0}: C_{n}^{1} \longrightarrow \operatorname{Ker} \mathfrak{E}, \\
e \longrightarrow\left(P_{0} e\right)(t)=c\left(\sum_{i=1}^{n} \int_{0}^{T}\left\langle e(s), \varphi_{i}(s)\right\rangle d s\right) \varphi_{i}(t),
\end{gathered}
$$

where $c=\left(\int_{0}^{T}\left\|e_{L}(s)\right\|_{0}^{2} d s\right)^{-2}$ is chosen in order to normalize $P_{0} e$.

$P_{0}$ is a projection on Ker $\mathfrak{E}$ and therefore,

$$
\forall e \in C_{n}^{1}: e=e_{1}+v
$$

where $e_{1} \in \operatorname{Ker} \mathfrak{E}$ and $v \in \hat{H}_{1}=\operatorname{Ker} \mathfrak{E}^{\perp}$. 
So, for all $e \in C_{n}^{1}, e=\sum_{i=1}^{n} \alpha_{i} \varphi_{i}+v$ where $\alpha_{i} \in \mathbb{R}$ and $v$ are such that

$$
\int_{0}^{T}\left\langle v(s), \varphi_{i}(s)\right\rangle d s=0, \quad i=1, \ldots, n
$$

Now, for the sake of notation's compactness, $\mathrm{\kappa}$ refers to $N G(e, \chi)$ and the projection $P_{1}$ is defined as follows:

$$
P_{1}: C_{n}^{0} \longrightarrow \operatorname{Im} \mathfrak{E}, \quad \mathrm{\aleph} \longrightarrow\left(P_{1} \aleph\right)(t)=\aleph(t)-\sum_{i=1}^{n}\left(\int_{0}^{T}\left\langle\aleph(s), \varphi_{i}(s)\right\rangle d s\right) \varphi_{i}(t)
$$

Therefore, for all $\mathrm{\kappa} \in C_{n}^{0}: \aleph(t)=\aleph_{1}(t)+\aleph_{2}(t)$, where $\aleph_{1}(t) \in \operatorname{Im} \mathfrak{E}$ and $\aleph_{2}(t) \in \hat{H}_{2}=$ $\operatorname{Im} \mathfrak{E}^{\perp}$.

Remark 2.8. The restriction of $\mathfrak{E}$ denoted by $\hat{\mathfrak{E}}: \hat{H}_{1} \rightarrow \operatorname{Im} \mathfrak{E}$ is invertible and the following operator: $K=\hat{\mathfrak{E}}^{-1}: P_{1} C_{n}^{0} \rightarrow\left(\mathrm{Id}-P_{0}\right) C_{n}^{1}$ is continuous.

Moreover, $\widehat{\mathfrak{E}} K=\operatorname{Id}$ on $\operatorname{Im} \mathfrak{E}$ and $K \hat{\mathfrak{E}}=\left(\mathrm{Id}-P_{0}\right)$ on $C_{n}^{1}$, where Id designs the identity operator.

Consequently and by Fredholm alternative, it comes to the following lemma.

Lemma 2.9. The function $e=K P_{1} \mathrm{~N}$ is a solution of the following system:

$$
\begin{gathered}
\mathfrak{E} e=P_{1} \aleph, \\
P_{0} K P_{1} \aleph=0,
\end{gathered}
$$

and each solution of system (2.9) is given by $e=\sum_{i=1}^{n} \alpha_{i} \varphi_{i}+K P_{1} \mathrm{~N}$, where $\alpha_{i} \in \mathbb{R}, i=$ $1, \ldots, n$, and $K P_{1} \kappa \in\left(\operatorname{Id}-P_{0}\right) C_{n}^{1}$.

Proof. The equation $\mathfrak{E} e=\mathrm{N}$ admits a solution if and only if $\left(\mathrm{Id}-P_{0}\right) \mathcal{N}=0([10])$. In this case, there exists a function $e$ solving (2.9) such that $P_{0} e(\mathrm{\aleph})=0$.

This first lemma allows us to propose a second one.

Lemma 2.10. Equation (2.9) is equivalent (equivalent in the meaning that each solution of (2.9) is a solution of (2.17) and reciprocally) to

$$
\mathfrak{E}\left(\sum_{i=1}^{n} \alpha_{i} \varphi_{i}+v\right)=\aleph\left(\sum_{i=1}^{n} \alpha_{i} \varphi_{i}+v, \chi\right), \quad \alpha_{i} \in \mathbb{R}, i=1, \ldots, n, v \in\left(\operatorname{Id}-P_{0}\right) C_{n}^{1}, \chi \in X
$$

Proof. Applying $P_{0}, K P_{1}$, and ( $\left.\mathrm{Id}-P_{0}\right)$ to the system (2.9), (2.17) is immediately obtained. 
It is now possible to deduce the following lemma.

LEMmA 2.11. The function $e$ is a solution of (2.17) if and only if $e=\sum_{i=1}^{n} \alpha_{i} \varphi_{i}+v$, where $\alpha_{i} \in \mathbb{R}$ and $v \in\left(\mathrm{Id}-P_{0}\right) C_{n}^{1}$ is solution of

$$
\begin{gathered}
v=K P_{1} \aleph\left(\sum_{i=1}^{n} \alpha_{i} \varphi_{i}+v, \chi\right), \\
0=\left(\mathrm{Id}-P_{1}\right) \curvearrowright\left(\sum_{i=1}^{n} \alpha_{i} \varphi_{i}+v, \chi\right) .
\end{gathered}
$$

Proof. Lemma 2.11 results directly from the application of the projections $P_{0}, P_{1}, K$, and the Fredholm alternative to (2.17).

Remark 2.12. Equation (2.18a) is called the auxiliary equation and (2.18b) is called the bifurcation equation.

2.3.1. Auxiliary equation analysis. The correction function $v$ modifies the linear solution in order to take into account the nonlinear behavior of the system. This function can be rewritten in the neighborhood of $e=0$ as

$$
v=\left(e(0)-\sum_{i=1}^{n} \alpha_{i} \varphi_{i}(0)\right)+w, \quad w \in\left(\operatorname{Id}-P_{0}\right) C_{n}^{1} .
$$

In order to analyze the existence of solutions of (2.18a), let us introduce a new operator:

$$
G:\left(\mathrm{Id}-P_{0}\right) C_{n}^{1} \times \mathbb{R}^{n} \times X \times \mathbb{R}^{n} \longrightarrow\left(\mathrm{Id}-P_{0}\right) C_{n}^{1}
$$

defined as

$$
G(w, \alpha, \chi, e(0))=\left(e(0)-\sum_{i=1}^{n} \alpha_{i} \varphi_{i}(0)\right)+w-K P_{1} \aleph\left(\sum_{i=1}^{n} \alpha_{i} \varphi_{i}+\left(e(0)-\sum_{i=1}^{n} \alpha_{i} \varphi_{i}(0)\right)+w, \chi\right) .
$$

This operator is defined by replacing (2.19) in (2.18a).

The analysis of the auxiliary equation is thus reduced to the analysis of the equation $G(w, \alpha, \chi, e(0))=0$.

From Assumptions 2.1 and 2.2, it can be deduced that $G$ satisfies the following statements:

(i) $G(0,0,0,0)=0$;

(ii) $G$ is $C^{2}$ with respect to $w$;

(iii) $D_{w} G(0,0,0,0)=\left.\operatorname{Id}\right|_{\left(\mathrm{Id}-P_{0}\right) C_{n}^{1}}$, where $D_{w}$ is the Frechet partial derivative of $G$ relatively to $w$.

Now, using the implicit function theorem, the following proposition is obtained.

Proposition 2.13. There exist a neighborhood $V_{w}$ of $w=0$ in $\left(\mathrm{Id}-P_{0}\right) C_{n}^{1}$, a neighborhood $V_{\alpha}$ of $\alpha=0$ in $\mathbb{R}^{n}$, a neighborhood $V_{\chi}$ of $\chi=0$ in $X$, and a neighborhood $V_{e(0)}$ of $e(0)=0$ in $\mathbb{R}^{n}$ such that for $(\alpha, \chi, e(0)) \in V_{w} \times V_{\chi} \times V_{e(0)}$, the auxiliary equation (2.18a) admits one 
and only one solution $w^{*}(\alpha, \chi, e(0)) \in V_{w}$ such that $w^{*}(0,0,0)=0$ if and only if $(\alpha, \chi, e(0))$ is a solution of bifurcation equation (2.18b).

Remark 2.14. As $w^{*}$ is uniquely determined in $V_{w}$, the number of solutions of (2.17) is exactly determined by the number of $\alpha=\left(\alpha_{1}, \ldots, \alpha_{n}\right)$ solutions of the bifurcation equation (2.18b). This is due to the fact that each solution of (2.17) can be written as

$$
e=\sum_{i=1}^{n} \alpha_{i} \varphi_{i}+\left(e(0)-\sum_{i=1}^{n} \alpha_{i} \varphi_{i}(0)\right)+w^{*}(\alpha, \chi, e(0))
$$

In fact, this formulation of our problem transforms the resolution of the infinite dimensional problem (2.17) into the resolution of two equations: the first one is the auxiliary equation and is in infinite dimension but has one and only one solution $w^{*}$ and the second one is resolved in $\mathbb{R}^{n}$ and thus is in finite dimension $(n)$.

2.3.2. Bifurcation equation analysis. As it was proved in the previous subsection that (2.18a) admits one and only one solution $w^{*}$, the resolution of the system $(2.18)$ is reduced to the bifurcation equation analysis $(2.18 \mathrm{~b})$, where $w^{*}$ is substituted to $w$. Thus system (2.18) admits at least one solution if and only if $\alpha$ satisfies the bifurcation (2.18b) which may be rewritten for $i=1, \ldots, n$ as

$$
\begin{aligned}
& I_{i}(\alpha, \chi, e(0)) \\
& =\int_{0}^{T}\left\langle\left(\gamma\left(\sum_{i=1}^{n} \alpha_{i} \varphi_{i}+\left(e(0)-\sum_{i=1}^{n} \alpha_{i} \varphi_{i}(0)\right)+w^{*}(\alpha, \chi, e(0)), \chi, t\right)\right), \varphi_{i}(t)\right\rangle d t=0, \\
& \Xi=\left(I_{1}, \ldots, I_{n}\right) .
\end{aligned}
$$

In the analysis of this system of the $n$ previous equations, at least two cases occur. Here, only two cases corresponding to Assumptions 2.1 and 2.2 are considered.

Case 1. There exist $\alpha_{0}, \chi_{0}$ and an arbitrary $e_{f}(0)$ in $V_{\alpha}, V_{\chi_{0}}$, and $V_{e(0)}$, respectively, such that

$$
\begin{array}{r}
\Xi\left(\alpha_{0}, \chi_{0}, e_{f}(0)\right)=0, \\
\nabla_{\alpha} \Xi\left(\alpha_{0}, \chi_{0}, e_{f}(0)\right) \text { (the Jacobian matrix of } \Xi \text { relatively to } \alpha \text { at } \\
\left.\left(\alpha_{0}, \chi_{0}, e_{f}(0)\right)\right) \text { is invertible. }
\end{array}
$$

The implicit function theorem ensures then that there exist a neighborhood $V_{\alpha_{0}}$ of $\alpha_{0}$ in $\mathbb{R}^{n}$, a neighborhood $V_{\chi_{0}}$ of $\chi_{0}$ in $X$, a neighborhood $V_{e_{f}(0)}$ of $e_{f}(0)$ in $\mathbb{R}^{n}$, and one and only one application $\alpha^{*}: v_{\chi_{0}} \times V_{e_{f}(0)} \rightarrow v_{\alpha_{0}}$ such that $\alpha^{*}\left(\chi_{0}, e_{f}(0)\right)=\alpha_{0}$ and $\Xi\left(\alpha^{*}\left(\chi, e_{f}(0)\right)\right.$, $\left.\chi, e_{f}(0)\right)=0$, for all $e_{f}(0) \in V_{e_{f}(0)}$, for all $\chi \in V_{\chi_{0}}$. 
Therefore, the problem (2.17) admits one and only one solution near $e_{L}$ which is denoted $e^{*}$ and given by

$$
e^{*}=\sum_{i=1}^{n} \alpha_{i}^{*}\left(\chi, e_{f}(0)\right) \varphi_{i}+\left(e(0)-\sum_{i=1}^{n} \alpha_{i}^{*}\left(\chi, e_{f}(0)\right) \varphi_{i}(0)\right)+w^{*}\left(\alpha^{*}\left(\chi, e_{f}(0)\right), \chi, e(0)\right) .
$$

Consequently, there is no bifurcation.

Case 2. the condition of the first case is not satisfied, that is $\exists v_{\alpha} \times v_{\chi} \times v_{e(0)} \subset V_{\alpha} \times V_{\chi} \times$ $V_{e(0)}$ such that for all $(\alpha, \chi, e(0)) \in v_{\alpha} \times v_{\chi} \times v_{e(0)}$

$$
\Xi(\alpha, \chi, e(0))=0 \Longrightarrow \nabla_{\alpha} \Xi(\alpha, \chi, e(0)) \text { is not invertible. }
$$

So, the resolution of bifurcation equations in $\mathbb{R}^{n}$ is based on numerical methods (see [11], e.g.).

In order to detail all the technical points of the proposed approach, some results concerning the case $n=1$ are presented hereafter.

2.4. Bifurcation analysis, case $n=1$. Let us consider the following system in $\mathbb{R}$ :

$$
\dot{e}=a(t) e+\gamma(e, \chi, t)
$$

where $t \in J=[0, T], e: J \rightarrow M \subset \mathbb{R}$ is the state and $M$ is a nonempty open set of $\mathbb{R} \cdot \chi: J \rightarrow$ $\Omega \subset \mathbb{R}$ is an external signal and $\Omega$ is a nonempty open set of $\mathbb{R} . a: J \longrightarrow \mathbb{R}$ is a continuous application. $\gamma: M \times \Omega \times J \rightarrow \mathbb{R}$ is an application which regroups all the nonlinearities and satisfies Assumptions 2.1 and 2.2. Moreover, the following assumption holds.

Assumption 2.15. There exist $\varphi(t)$ a Lebesgue integrable and positive function such that

$$
\int_{0}^{T}\left|\frac{\partial^{2} \gamma}{\partial e^{2}}(0,0, t)\right| d t \geq \int_{0}^{T} \varphi(t) d t
$$

Let us recall that the purpose is to analyze the number of solutions of (2.27) in the neighborhood of a nontrivial solution $e_{L}$ of the related linear system:

$$
\dot{e}=a(t) e .
$$

2.4.1. Abstract problem formulation. For the sake of notation simplicity, $C^{0}$ and $C^{1}$, respectively, stand for $C_{0}^{0}(J, \mathbb{R})$ and $C_{0}^{1}(J, \mathbb{R})$.

$X \subset C_{0}^{0}(J, \Omega)$ is the set of continuous external signal $\chi$.

Some results are first recalled.

Proposition 2.16 [9]. A general solution of linear (2.29) is given by

$$
e_{L}(t)=\phi(t) e(0), \quad t \in J,
$$

where $\phi(t)=\exp \left(\int_{0}^{t} a(s) d s\right)$. 
Proposition 2.17 [9]. A function $e \in C^{1}$ is a solution of the nonlinear problem (2.27) if and only if it is a solution of the following equation:

$$
e(t)=e_{L}(t)+\int_{0}^{t} \phi(t) \phi^{-1}(s) \gamma(e(s), \chi(s), s) d s .
$$

The linear operator $L$ is defined as

$$
\begin{gathered}
L: C^{1} \longrightarrow C^{0}, \\
(L e)(t)=\dot{e}(t)-a(t) e(t),
\end{gathered}
$$

and the Nemystskii nonlinear operator [10] $N$ is given by

$$
N: C^{1} \times X \rightarrow C^{0} \quad \text { such that } N(e, \chi)(t)=\gamma(e(t), \chi(t), t) .
$$

Consequently, we obtain the following proposition.

Proposition 2.18. Equation (2.27) is equivalent (equivalent in the meaning that each formal solution of (2.34) is an abstract solution of (2.27) and, reciprocally) to

$$
L e=N(e, \chi), \quad e \in C^{1}, \chi \in X .
$$

2.5. Problem analysis. As $e(0)$ is different from 0 and is not fixed, $L$ satisfies the following statements.

Proposition 2.19. (i) $L$ is a linear continuous and bounded operator.

(ii) $\operatorname{dim} \operatorname{Ker} L=\operatorname{codim} \operatorname{Im} L=1$ and $L$ is a Fredholm operator of index 0 .

(iii) There exists $H_{1}: C^{1}=\operatorname{Ker} L \oplus H_{1}$, and there exist $H_{2}: C^{0}=\operatorname{Im} L \oplus H_{2}$ such that

$$
\begin{gathered}
\forall e \in C^{1}, \quad \exists \alpha \in \mathbb{R}, \quad \exists v \in H_{1}: e=\alpha e_{L}+v, \\
\forall h \in C^{0}, \quad \exists h_{1} \in \operatorname{Im} L, \quad \exists h_{2} \in H_{2}: h=h_{1}+h_{2} .
\end{gathered}
$$

Proof. (i) Results from the definition of $L$.

(ii) $\operatorname{Ker} L$ is spanned by $\phi(t)$, thus $\operatorname{dim} \operatorname{Ker} L=1$ and from the Fredholm alternative, the problem $L e=N(e, \chi)$ admits a solution if and only if

$$
\int_{0}^{T} N(e, \chi)(t) e_{L}(t) d t=0 .
$$

So $\operatorname{dim} \operatorname{Ker} L=\operatorname{codim} \operatorname{Im} L=1$ and so Index $L=0$.

(iii) The projection over $P_{0}$ is defined as

$$
\begin{gathered}
P_{0}: C^{1} \longrightarrow \operatorname{Ker} L \\
e \longrightarrow\left(P_{0} e\right)(t)=c\left(\int_{0}^{T} e(s) e_{L}(s) d s\right) e_{L}(t),
\end{gathered}
$$

where $c=\left(\int_{0}^{T}\left|e_{L}(s)\right|^{2} d s\right)^{-2}$. 
Therefore, for all $e \in C^{1}: e=\alpha e_{L}+v$, where $\alpha \in \mathbb{R}$ and $v$ is such that

$$
\int_{0}^{T} v(s) e_{L}(s) d s=0
$$

Let us denote (similarly to the case $n>1) N(e, \chi)=\kappa$ and let us define the projection $P_{1}$ as

$$
\begin{gathered}
P_{1}: C^{0} \longrightarrow \operatorname{Im} L, \\
\aleph \longrightarrow\left(P_{1} \aleph\right)(t)=\aleph(t)-\left(\int_{0}^{T} \aleph(s) e_{L}(s) d s\right) e_{L}(t) .
\end{gathered}
$$

Therefore, for all $\mathrm{\kappa} \in C^{0}: \mathrm{\kappa}(t)=\mathrm{\aleph}_{1}(t)+\mathrm{\aleph}_{2}(t)$, where $\mathrm{\aleph}_{1}(t) \in \operatorname{Im} L$ and $\mathrm{\aleph}_{2}(t) \in H_{2}=$ $\operatorname{Im} L^{\perp}$.

Remark 2.20. The restriction of $L$ denoted by $\widehat{L}: H_{1} \longrightarrow \operatorname{Im} L$ is invertible.

The operator $k=\widehat{L}^{-1}: P_{1} C^{0} \rightarrow\left(\operatorname{Id}-P_{0}\right) C^{1}$ is continuous, $\hat{L} k=\operatorname{Id}$ on $\operatorname{Im} L$ and $k \widehat{L}=$ $\left(\mathrm{Id}-\mathrm{P}_{0}\right)$ on $C^{1}$.

Consequently and using the Fredholm alternative, the following lemma is obtained.

Lemma 2.21. The function $e=K P_{1} \mathrm{~N}$ is a solution of the following system:

$$
\begin{gathered}
L e=P_{1} \ltimes, \\
P_{0} k P_{1} \ltimes=0,
\end{gathered}
$$

and each solution of the system (2.34) is given by $e=\alpha e_{L}+k P_{1} \kappa$, where $\alpha \in \mathbb{R}$ and $k P_{1} \mathrm{~N} \in$ $\left(\mathrm{Id}-P_{0}\right) C^{1}$.

Using this lemma, it comes to the following one.

LEMma 2.22. Equation (2.34) is equivalent (equivalent in the meaning that each solution of (2.34) is a solution of (2.41) and reciprocally) to

$$
L\left(\alpha e_{L}+v\right)=N\left(\alpha e_{L}+v, \chi\right), \quad \alpha \in \mathbb{R}, v \in\left(\mathrm{Id}-P_{0}\right) C^{1}, \chi \in X .
$$

Applying the projections $P_{0}, P_{1}, k$, and the Fredholm alternative to (2.41), we obtain the following lemma.

LEMMA 2.23. The function $e$ is a solution of (2.41) if and only if $e=\alpha e_{L}+v$, where $(\alpha, v) \in$ $\mathbb{R} \times\left(\mathrm{Id}-\mathrm{P}_{0}\right) \mathrm{C}^{1}$ is a solution of

$$
\begin{gathered}
v=K P_{1} N\left(\alpha e_{L}+v, \chi\right), \\
0=\left(\mathrm{Id}-P_{1}\right) N\left(\alpha e_{L}+v, \chi\right) .
\end{gathered}
$$

2.5.1. Auxiliary equation analysis. Recalling that the correction function $v$ modifies the linear solution so as to take into account the nonlinear behavior of the system, it can be rewritten in the neighborhood of $e=0$ as

$$
v=(1-\alpha) e(0)+w, \quad w \in\left(\mathrm{Id}-P_{0}\right) C^{1} .
$$


In order to analyze the existence of solutions of (2.42a), a new operator (noted also by $G$ as for the case $n>1$ ) is introduced:

$$
G:\left(\mathrm{Id}-P_{0}\right) C^{1} \times \mathbb{R} \times X \times \mathbb{R} \longrightarrow\left(\mathrm{Id}-P_{0}\right) C^{1} .
$$

$G$ is defined as

$$
G(w, \alpha, \chi, e(0))=(1-\alpha) e(0)+w-k P_{1} N\left(\alpha e_{L}+(1-\alpha) e(0)+w, \chi\right) .
$$

Analyzing the auxiliary equation is equivalent to analyzing the equation $G(w, \alpha, \chi, e(0))=$ 0 .

From Assumptions 2.1 and 2.2, it can be deduced that $G$ satisfies the following statements:

(i) $G(0,0,0,0)=0$;

(ii) $G$ is $C^{2}$ with respect to $w$;

(iii) $D_{w} G(0,0,0,0)=\left.\operatorname{Id}\right|_{\left(\mathrm{Id}-P_{0}\right) C^{1}}$.

Now, using the implicit function theorem, it comes to the following proposition.

Proposition 2.24. There exist a neighborhood $V_{w}$ of $w=0$ in $\left(\mathrm{Id}-P_{0}\right) C^{1}$, a neighborhood $V_{\alpha}$ of $\alpha=0$ in $\mathbb{R}$, a neighborhood $V_{\chi}$ of $\chi=0$ in $X$, and a neighborhood $V_{e(0)}$ of $e(0)=0$ in $\mathbb{R}$ such that for $(\alpha, \chi, e(0)) \in V_{w} \times V_{\chi} \times V_{e(0)}$, the auxiliary equation (2.42a) admits one and only one solution $w^{*}(\alpha, \chi, e(0)) \in V_{w}$ such that $w^{*}(0,0,0)=0$ if and only if $(\alpha, \chi, e(0))$ is the solution of the bifurcation equation (2.42b).

Remark 2.25. As $w^{*}$ is uniquely determined in $V_{w}$, the number of solutions of (2.41) is exactly determined by the number of $\alpha$ solutions of the bifurcation equation (2.42b). The resolution of bifurcation equation will be done in $\mathbb{R}$.

2.5.2. Bifurcation equation analysis. It was highlighted in the previous subsection that the system (2.42) admits a solution if and only if $\alpha$ satisfies the bifurcation equation and in the scalar case, $(2.42 \mathrm{~b})$ can be rewritten as follows:

$$
I_{1}(\alpha, \chi, e(0))=\int_{0}^{T} \gamma\left(\alpha e_{L}+(1-\alpha) e(0)+w^{*}(\alpha, \chi, e(0)), \chi, t\right) e_{L}(t) d t=0 .
$$

For the sake of simplicity, $I$ stands for $I_{1}$.

Case 1. There exist $\alpha_{0}, \chi_{0}$ and an arbitrary $e_{f}(0)$ in $V_{\alpha_{0}}, V_{\chi_{0}}$, and $V_{e(0)}$, respectively, such that

$$
\begin{gathered}
I\left(\alpha_{0}, \chi_{0}, e_{f}(0)\right)=0, \\
\frac{\partial I}{\partial \alpha}\left(\alpha_{0}, \chi_{0}, e_{f}(0)\right) \neq 0 .
\end{gathered}
$$

The implicit function theorem ensures that there exist a neighborhood $V_{\alpha_{0}}$ of $\alpha_{0}$ in $\mathbb{R}$, a neighborhood $V_{\chi_{0}}$ of $\chi_{0}$ in $X$, a neighborhood $V_{e_{f}(0)}$ of $e_{f}(0)$ in $\mathbb{R}$, and a unique application $\alpha^{*}: v_{\chi_{0}} \times V_{e_{f}(0)} \rightarrow v_{\alpha_{0}}$ such that $\alpha^{*}\left(\chi_{0}, e_{f}(0)\right)=\alpha_{0}$ and $I\left(\alpha^{*}\left(\chi, e_{f}(0)\right), \chi, e_{f}(0)\right)=0$, for all $e_{f}(0) \in V_{e_{f}(0)}$, for all $\chi \in V_{\chi_{0}}$. 
In this case, the problem (2.41) admits one and only one solution $e^{*}=\alpha^{*}(\chi, e(0)) e_{L}+$ $\left(1-\alpha^{*}(\chi, e(0))\right) e(0)+w^{*}\left(\alpha^{*}(\chi, e(0)), \chi, e(0)\right)$ near $e_{L}$ and consequently there is no bifurcation.

Case 2. The condition of the first case is not satisfied, then $\exists v_{\alpha} \times v_{\chi} \times v_{e(0)} \subset V_{\alpha} \times V_{\chi} \times$ $V_{e(0)}$ such that for all $(\alpha, \chi, e(0)) \in v_{\alpha} \times v_{\chi} \times v_{e(0)}$,

$$
I(\alpha, \chi, e(0))=0 \Longrightarrow \frac{\partial I}{\partial \alpha}(\alpha, \chi, e(0))=0
$$

because of Assumption 2.15, it comes that

$$
\frac{\partial^{2} I}{\partial \alpha^{2}}(0,0,0) \neq 0
$$

From (2.48), (2.49), and the implicit function theorem, there exist a neighborhood $\tilde{v}_{\alpha} \subset v_{\alpha}$ of $\alpha=0$ in $\mathbb{R}$, a neighborhood $\tilde{v}_{\chi} \subset v_{\chi}$ of $\chi=0$ in $X$, a neighborhood $\tilde{v}_{e(0)} \subset$ $v_{e(0)}$ in $\mathbb{R}$, and a unique application $\alpha^{* *}: \tilde{v}_{\chi} \times \tilde{v}_{e(0)} \rightarrow \tilde{v}_{\alpha}$ such that $\alpha^{* *}(0,0)=0$ and $(\partial I / \partial \alpha)\left(\alpha^{* *}(\chi, e(0)), \chi, e(0)\right)=0$, for all $\chi \in \tilde{v}_{\chi}$, for all $e(0) \in \tilde{v}_{e(0)}$.

Therefore, considering the quadratic Taylor expansion of $I(\cdot, \chi, e(0))$ near $\alpha^{* *}$,

$$
\begin{aligned}
I(\alpha, \chi, e(0))= & I\left(\alpha^{* *}(\chi, e(0)), \chi, e(0)\right)+\frac{1}{2} \frac{\partial^{2} I}{\partial \alpha^{2}}\left(\alpha^{* *}(\chi, e(0)), \chi, e(0)\right)\left(\alpha-\alpha^{* *}(\chi, e(0))\right)^{2} \\
& +O\left(\left|\alpha-\alpha^{* *}(\chi, e(0))\right|^{2}\right)
\end{aligned}
$$

when $\alpha \rightarrow \alpha^{* *}(\chi, e(0))$.

For the sake of compactness, $\alpha^{*}$ stands for $\alpha^{*}(\chi, e(0))$.

The following equation has to be solved:

$$
I(\alpha, \chi, e(0))=o\left(\left|\alpha-\alpha^{* *}\right|^{3}\right)
$$

It is assumed that for $\tilde{v}_{\alpha}$ sufficiently small around $0,\left(\partial^{2} I / \partial \alpha^{2}\right)\left(\alpha^{* *}, \chi, e(0)\right)$ and $\left(\partial^{2} I /\right.$ $\left.\partial \alpha^{2}\right)(0, \chi, e(0))$ have the same sign.

Moreover, we know that if $\left(\partial^{2} I / \partial \alpha^{2}\right)(0, \chi, e(0))>0$, then $\alpha^{* *}$ is a minimum for $I(\cdot, \chi$, $e(0))$. Similarly if $\left(\partial^{2} I / \partial \alpha^{2}\right)(0, \chi, e(0))<0$ then $\alpha^{* *}$ is a maximum for $I(\cdot, \chi, e(0))$.

Consequently, the following five possibilities appear:

(i) when $\left(\partial^{2} I / \partial \alpha^{2}\right)(0, \chi, e(0))>0$ and $I\left(\alpha^{* *}, \chi, e(0)\right)<0$, then $(2.51)$ has two solutions in the neighborhood of $\alpha^{* *}$;

(ii) when $\left(\partial^{2} I / \partial \alpha^{2}\right)(0, \chi, e(0))<0$ and $I\left(\alpha^{* *}, \chi, e(0)\right)>0$, then $(2.51)$ has two solutions in the neighborhood of $\alpha^{* *}$;

(iii) when $\left(\partial^{2} I / \partial \alpha^{2}\right)(0, \chi, e(0))>0$ and $I\left(\alpha^{* *}, \chi, e(0)\right)>0$, then $(2.51)$ has no solution in the neighborhood of $\alpha^{* *}$;

(iv) when $\left(\partial^{2} I / \partial \alpha^{2}\right)(0, \chi, e(0))<0$ and $I\left(\alpha^{* *}, \chi, e(0)\right)<0$, then $(2.51)$ has no solution in the neighborhood of $\alpha^{* *}$.

(v) when $I\left(\alpha^{* *}, \chi, e(0)\right)=0$, then $(2.51)$ has one unique solution $\alpha^{* *}$. 
It can be noticed that this method using the study of some functions signs is specific to the case $n=1$, this justifies the addition of Assumption 2.15.

In order to synthesize all the previous results, the following that we call singular operator is introduced:

$$
\begin{gathered}
S: \tilde{v}_{\chi} \times \tilde{v}_{e(0)} \longrightarrow \mathbb{R}, \\
S(\chi, e(0))=I\left(\alpha^{* *}, \chi, e(0)\right) \frac{\partial^{2} I}{\partial \alpha^{2}}(0, \chi, e(0)) .
\end{gathered}
$$

The bifurcation set Bif $:=\left\{(\chi, e(0)) \in \tilde{v}_{\chi} \times \tilde{v}_{e(0)}: S(\chi, e(0))=0\right\}$ characterizes the transient manifold such that the solution number changes with respect to $\chi$ and $e(0)$.

And naturally, we set

$$
\begin{aligned}
& W^{-}=\left\{(\chi, e(0)) \in \tilde{v}_{\chi} \times \tilde{v}_{e(0)}: S(\chi, e(0))<0\right\} \\
& W^{+}=\left\{(\chi, e(0)) \in \tilde{v}_{e(0)} \times \tilde{v}_{e(0)}: S(\chi, e(0))>0\right\} .
\end{aligned}
$$

In the next theorem, the solution's bifurcation generated by the move from Bif to $W^{+}$or $W^{-}$is highlighted.

Theorem 2.26. Under Assumptions 2.1, 2.2, and 2.15, there exist a neighborhood $\tilde{v}_{w}$ of $w=0$ in $C^{1}$, a neighborhood $\widetilde{v}_{\alpha}$ of $\alpha=0$ in $\mathbb{R}$, a neighborhood $\widetilde{v}_{\chi}$ of $\chi=0$ in $X$, and $a$ neighborhood $\tilde{v}_{e(0)}$ of 0 in $\mathbb{R}$ such that if

(1) $(\chi, e(0)) \in W^{-}$, then problem (2.27) admits two distinct solutions which bifurcate from $e_{L}$.

(2) $(\chi, e(0)) \in$ Bif, then problem (2.27) admits one and only solution in the neighborhood of $e_{L}$.

(3) $(\chi, e(0)) \in W^{+}$, then problem (2.27) has no solution in the neighborhood of $e_{L}$.

This theorem has many direct and important applications in systems theory, for example, the robustness and the validity analysis of linear control by gain schedule applied to nonlinear systems. A natural dual case of the previous one is the case of linear observer applied to nonlinear systems. Hereafter, a very simple academic application is first given. In this example, all the computational aspects of this analysis will be detailed. Moreover, a nonlinear corrective term is proposed in order to overcome the bifurcation submanifold. Finally, the study of reduced observer design for practical and well-known Lagrangian system ends the observer analysis section in order to highlight the practical engineering aspect of our analysis.

\section{Observer analysis}

3.1. Problem statement. Considering the following nonlinear system:

$$
\begin{aligned}
& \dot{\chi}=\bar{a} \chi+\Gamma_{[2]}(\chi), \\
& y=c \chi+h_{[2]}(\chi),
\end{aligned}
$$

with $\chi \in \mathbb{R}$ is the state, $y \in \mathbb{R}$ is the output, and $\bar{a}$ and $c$ are some constants different from 0 and functions $\Gamma_{[2]}(\cdot)$ and $h_{[2]}(\cdot)$ are at least quadratic in $\chi$. 
The linear approximation of $(3.1)$ is

$$
\begin{aligned}
& \dot{\chi}=\bar{a} \chi, \\
& y=c \chi .
\end{aligned}
$$

For system (3.2), there exist many observer schemes. Hereafter, the classical Luemberger observer is considered:

$$
\begin{gathered}
\dot{\hat{\chi}}=\bar{a} \hat{\chi}+k(y-\hat{y}), \\
\hat{y}=c \hat{\chi} .
\end{gathered}
$$

It is well known that the observation error $e=\chi-\hat{\chi}$ with respect to the linear approximation is given by

$$
\dot{e}=(\bar{a}-k c) e:=a e .
$$

Consequently, the observer gain $k \in \mathbb{R}$ is computed such that $e$ is exponentially stable.

Unfortunately, the real system is generally not the linear one (3.2) but the nonlinear one (3.1). Consequently, the real observation error dynamic is

$$
\dot{e}=a e+\Gamma_{[2]}(\chi)-k h_{[2]}(\chi) .
$$

From Theorem 2.26, the question of the relevance to design a linear observer based on the linear approximation (3.2) for the nonlinear system (3.1) is of prior importance. In practice, the gain $k$ is increased in order to crush the nonlinearity's effect. In fact, this strategy has two negative effects: first of all, increasing $k$ is equivalent to decrease the filtering effects of the observer and secondly this does not guarantee to avoid the solution's bifurcation.

Remark 3.1. $\gamma^{\prime}(e, \chi, t)=\Gamma_{[2]}(\chi)-\Gamma_{[2]}(\chi-e)-k\left(h_{[2]}(\chi)-h_{[2]}(\chi-e)\right)$ the nonlinear part of the observation error dynamics corresponding to use the linear observer (3.3) in order to observe (3.1) verifies Assumptions 2.1, 2.2, and 2.15. Consequently, from Theorem 2.26 , the solution's bifurcation problem may appear in the usual observer design if $\chi$ and $e(0)$ cross a certain manifold.

In order to avoid this problem, the following observer scheme is proposed:

$$
\begin{gathered}
\dot{\hat{\chi}}=\bar{a} \hat{\chi}+k(y-\hat{y})+\theta_{[2]}\left(\hat{\chi}, y-\hat{y}+d+h_{[2]}(\hat{\chi})\right), \\
\hat{y}=c \hat{\chi}+h_{[2]}(\tilde{\chi}),
\end{gathered}
$$

where $d \in \mathbb{R}$ is an acting parameter and $\theta_{[2]}$ is a quadratic correcting term (only depending on known variables).

Thus, the following new observation error dynamics is obtained:

$$
\dot{e}=a e+\tilde{\gamma}(e, \chi, d, t)
$$

where $\tilde{\gamma}(e, \chi, d, t)=\Gamma_{[2]}(\chi)-k\left(h_{[2]}(\chi)-h_{[2]}(\chi-e)\right)-\theta_{[2]}\left(\chi-e, c e+d+h_{[2]}(\chi)\right)$ is the nonlinear term of (3.7) ( $\theta_{[2]}$ will be designed with respect to Theorem 2.26). This design 
is realized in order to overcome the bifurcation and to locally obtain a unique solution of (3.7) close to $e_{L}$ for any $e(0)$.

In order to take into account the correction term $d$, the related nonlinear operator $\tilde{N}$ is modified as

$$
\tilde{N}: C^{1} \times X \times \mathbb{R} \longrightarrow C^{0},
$$

where $\tilde{N}(e, \chi, d)(t)=\tilde{\gamma}(e, \chi, d, t)$. Consequently, the following corollary holds.

Corollary 3.2. Equation (3.7) is equivalent (equivalent in the meaning that each solution of (3.9) is solution of problem (3.7) and reciprocally) to

$$
\text { Le }=\tilde{N}(e, \chi, d), \quad \text { where } e \in C^{1}, \chi \in X, d \in \mathbb{R} .
$$

Let us note that the projections $P_{0}, P_{1}$, and $k$ are defined as in Section 2 but relatively to the operator $\tilde{N}$. Lemma 2.22 thus becomes the following corollary.

Corollary 3.3. Equation (3.9) is equivalent (equivalent in the meaning that each solution of (3.10) is a solution of (3.9) and reciprocally) to

$$
L\left(\alpha e_{L}+\tilde{v}\right)=\tilde{N}\left(\alpha e_{L}+\tilde{v}, \chi, d\right), \quad \alpha \in \mathbb{R}, \tilde{v} \in\left(I d-P_{0}\right) C^{1}, \chi \in X, d \in \mathbb{R}
$$

Similarly, Lemma 2.23 becomes the following corollary.

Corollary 3.4. The function $e$ is a solution of (3.10) if and only if $e=\alpha e_{L}+\tilde{v}$, where $(\alpha, v, d) \in \mathbb{R} \times\left(\mathrm{Id}-P_{0}\right) C^{1} \times \mathbb{R}$ is a solution of

$$
\begin{gathered}
\tilde{v}=K P_{1} \tilde{N}\left(\alpha e_{L}+\tilde{v}, \chi, d\right), \\
0=\left(\operatorname{Id}-P_{1}\right) \tilde{N}\left(\alpha e_{L}+\tilde{v}, \chi, d\right) .
\end{gathered}
$$

Naturally, from the same analysis as in Section 2 (reported in Appendix A), a new singularity operator is defined over $\bar{v}_{\chi} \times \bar{v}_{e(0)} \times \bar{v}_{d} \subset V_{\chi} \times V_{e(0)} \times V_{d}$ as

$$
\begin{gathered}
s: \bar{v}_{\chi} \times \bar{v}_{e(0)} \times \bar{v}_{d} \longrightarrow \mathbb{R}, \\
s(\chi, e(0), d)=\widetilde{I}\left(\hat{\alpha}^{* *}(\chi, e(0), d), \chi, e(0), d\right) \cdot \frac{\partial^{2} \tilde{I}}{\partial \alpha^{2}}(0, \chi, e(0), d) .
\end{gathered}
$$

The bifurcation set is modified as $\widetilde{B}$ if $:=\left\{(\chi, e(0), d) \in \bar{v}_{3} \times \bar{v}_{4} \times \bar{v}_{5}: s(\chi, e(0), d)=0\right\}$ and $\widetilde{W}^{-}$and $\widetilde{W}^{+}$are defined as

$$
\begin{aligned}
& \widetilde{W}^{-}=\left\{(\chi, e(0), d) \in \bar{v}_{3} \times \bar{v}_{4} \times \bar{v}_{5}: s(\chi, e(0), d)<0\right\}, \\
& \widetilde{W}^{+}=\left\{(\chi, e(0), d) \in \bar{v}_{3} \times \bar{v}_{4} \times \bar{v}_{5}: s(\chi, e(0), d)>0\right\} .
\end{aligned}
$$

This allows us to propose the following theorem.

Theorem 3.5. Under Assumptions 2.1, 2.2, 2.15 relatively to $\tilde{\gamma}$, there exist a neighborhood $\bar{v}_{\tilde{w}}$ of $\widetilde{w}=0$ in $C^{1}$, a neighborhood $\bar{v}_{\alpha}$ of $\alpha=0$ in $\mathbb{R}$, a neighborhood $\bar{v}_{\chi}$ of $\chi=0$ in $X, a$ 
neighborhood $\bar{v}_{e(0)}$ of $e(0)=0$ in $\mathbb{R}$, and a neighborhood $\bar{v}_{d}$ of $d=0$ in $\mathbb{R}$ such that

(1) if $(\chi, e(0), d) \in \widetilde{W}^{+}$, then problem (3.7) admits two distinct solutions which bifurcate from $e_{L}$;

(2) if $(\chi, e(0), d) \in \widetilde{B}$ if, then problem (3.7) admits a unique solution in the neighborhood of $e_{L}$;

(3) if $(\chi, e(0), d) \in \widetilde{W}^{-}$, then problem (3.7) has no solution in the neighborhood of $e_{L}$.

In order to reach the bifurcation set $\tilde{B}$ if when the dynamics are originally in $W^{+}$for example, an action on $d$ is used to obtain one and only one solution of the observation error (3.7) near the linear one.

As the function $s(\chi, e(0), \cdot)$ is continuous on $J$, the following assumption is introduced. Assumption 3.6. There exist $\left.d_{1}, d_{2} \in\right] \eta_{1}, \eta_{2}\left[\subset J\right.$ such that $s\left(\chi, e(0), d_{1}\right) s\left(\chi, e(0), d_{2}\right)<0$, for all $\chi \in \bar{v}_{\chi}$, for all $e(0) \in \bar{v}_{e(0)}$.

Assumptions 2.1, 2.2, 2.15, and 3.6 are sufficient to state that the observation error equation has a unique solution as it is asserted in the next proposition.

Proposition 3.7. Under Assumptions 2.1, 2.2, 2.15 (relatively to $\tilde{\gamma}$ ) and Assumption 3.6, the system (3.7) admits one and only one solution near $e_{L}$, for all $(\chi, e(0)) \in \bar{v}_{\chi} \times \bar{v}_{e(0)}$.

Proof. Under Assumptions 2.1, 2.2, 2.15, and 3.6, the intermediate value theorem applied to $s(\chi, e(0), \cdot)$ implies that there exists a correction term $\tilde{d} \in] d_{1}, d_{2}[$, such that $s(\chi, e(0), \tilde{d})$ $=0$, for all $(\chi, e(0)) \in \bar{v}_{\chi} \times \bar{v}_{e(0)}$, and so, by Theorem 3.5, there exists one unique solution of (3.7).

\section{Numerical examples}

Consider the following nonlinear one dimensional system defined as

$$
\begin{gathered}
\dot{\chi}=-0.1 \chi+0.1 \chi^{2}, \\
y=\chi+0.3 \chi^{2},
\end{gathered}
$$

where $\chi:[0,1] \rightarrow \mathbb{R}$ is the state and $y:[0,1] \rightarrow \mathbb{R}$ is the output.

An observer scheme which does not take into account the solution's bifurcation problem is first considered:

$$
\begin{gathered}
\dot{\hat{\chi}}=-0.1 \hat{\chi}+0.2(y-\hat{y})+0.1 \hat{\chi}^{2}, \\
\hat{y}=\hat{\chi}+0.3 \hat{\chi}^{2} .
\end{gathered}
$$

Thus, the observation error equation is

$$
\dot{e}=-0.3 e+\gamma(e, \chi)
$$

where $\gamma(e, \chi)=0.04\left(2 \chi e+e^{2}\right)$.

4.1. Bifurcation computation. It can be noticed that $\gamma$ satisfies Assumptions 2.1, 2.2, and 2.15. Thus, from Theorem 2.26, a solution's bifurcation may appear. The aim is to 
determine, if there are zero, one, or two solutions closed to $e_{L}$, the solution of the linear corresponding to the system $\dot{e}=-0.3 e$.

The Galerkin method [11] is an efficient tool for computing solutions when the implicit function theorem is claimed for solving formal equations. Let us introduce the following notations:

(i) $e_{h}$, the approximation of the state $e$ given by $e_{h_{L}}(t)=(1-0.3 t) e(0)$,

(ii) $\chi_{h}$, the approximation of the entry $\chi$ given by $\chi_{h}(t)=0.2-3 t$,

(iii) $v_{h}$, the approximation of $v$ given by $v_{h}(0)=(1-\alpha) e(0)$.

Consequently, after a simple computation, the approximation of $w, w_{h}$ at order one is given by $w_{h}(t)=-3 \alpha a t$, where $a$ is a real parameter.

In the first step, the coefficient $a$ which characterizes $w^{*}$ is computed. The computation is based on the resolution of the approximation at order one of the approximated auxiliary equation given by

$$
G_{a}\left(w_{h}, \alpha, \chi_{h}, e(0)\right):=G\left(w_{h}, \alpha, \chi_{h}, e(0)\right)=0 .
$$

We find $a=(1 / 25) e(0)(2(0.2)-e(0) / \alpha(-3))$.

Replacing this value in the bifurcation equation, it comes that

$$
I_{a}\left(\alpha, \chi_{h}, e(0)\right):=I\left(\alpha, \chi_{h}, e(0)\right)=0 .
$$

(See Appendix B for the computation of $G_{a}\left(w_{h}, \alpha, \chi_{h}, e(0)\right)$ and $I_{a}\left(\alpha, \chi_{h}, e(0)\right)$.)

This equation has two solutions:

$$
\begin{aligned}
& \alpha_{1}=-\frac{2}{2325} \frac{155 e(0)^{2}-10425-6062 e(0)-25 \sqrt{\left(173889-21450 e(0)^{2}+8580 e(0)\right)}}{e(0)}, \\
& \alpha_{2}=-\frac{2}{2325} \frac{155 e(0)^{2}-10425-6062 e(0)+25 \sqrt{\left(173889-21450 e(0)^{2}+8580 e(0)\right)}}{e(0)} .
\end{aligned}
$$

So, $\alpha_{1}$ and $\alpha_{2}$ correspond to two solutions of the observation error dynamics (4.3) given by

$$
\begin{aligned}
& e_{1}=\alpha_{1} e_{L}+\left(1-\alpha_{1}\right) e(0)+w^{*}\left(\alpha_{1}, \chi, e(0)\right), \\
& e_{2}=\alpha_{2} e_{L}+\left(1-\alpha_{2}\right) e(0)+w^{*}\left(\alpha_{2}, \chi, e(0)\right)
\end{aligned}
$$

which bifurcate from the expected linear solution $e_{L}(t)=\exp (-0.3 t) e(0)$.

Now, plotting $e_{L}$ (Figure 4.1), $e_{1}$ (Figure 4.2), and $e_{2}$ (Figure 4.3) in function of the initial condition $e(0)$ defined in $[-0.1,0.1]$ and with respect to the time $t$ defined in $[0,0.1]$, it can be seen that a solution's bifurcation appears. 
18 Lyapunov-Schmidt method and observer analysis

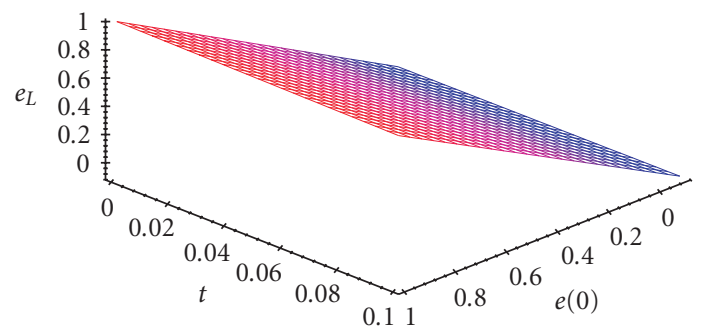

Figure 4.1

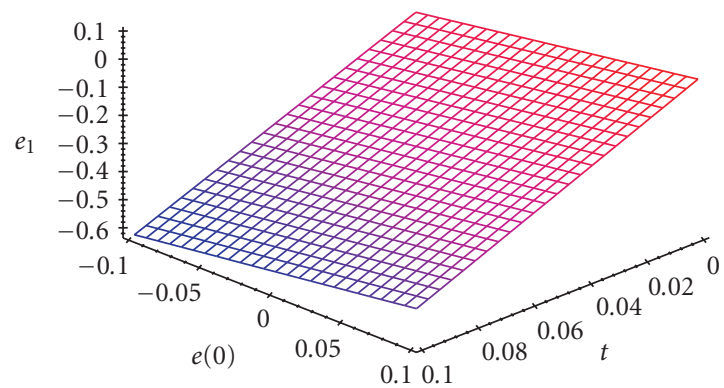

Figure 4.2

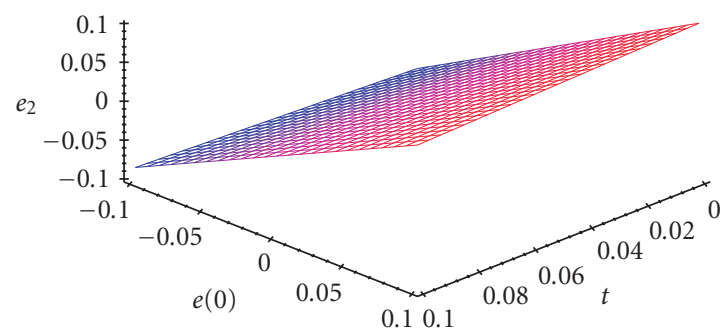

Figure 4.3

4.2. Bifurcation correction. In order to eliminate the bifurcation, the new type of observer introduced in (3.6) is used:

$$
\begin{gathered}
\dot{\hat{\chi}}=-0.1 \hat{\chi}+0.2(y-\hat{y})+0.4 \hat{\chi}^{2}\left(y-\hat{y}+d+0.3 \hat{\chi}^{2}\right)^{2}, \\
\hat{y}=\hat{\chi}+0.3 \hat{\chi}^{2} .
\end{gathered}
$$

The corresponding observation error is given by

$$
\dot{e}=-0.3 e+\hat{\gamma}(e, \chi, d),
$$

where $\hat{\gamma}(e, \chi, d)=0.1 \chi^{2}-0.06\left(-e^{2}+2 \chi e\right)+0.4(\chi-e)^{2}\left(e+d+0.3 \chi^{2}\right)$.

So, for the same entry $\chi$, the approximated auxiliary equation becomes

$$
G_{b}\left(w_{h}, \alpha, \chi_{h}, e(0), d\right):=G\left(w_{h}, \alpha, \chi_{h}, e(0), d\right)=0 .
$$


The resolution at order one of $G\left(w_{h}, \alpha, \chi_{h}, e(0), d\right)=0$ gives

$$
a=-\frac{1}{18750} \frac{2875 e(0)^{2}+19-620 e(0)-500 d+2500 e(0)}{\alpha} .
$$

Replacing $a$ in the corresponding bifurcation equation gives

$$
I_{b}(\alpha, \chi, e(0), d):=I(\alpha, \chi, e(0), d)=0 .
$$

(See Appendix $\mathrm{C}$ for the computation of $G_{b}\left(w_{h}, \alpha, \chi_{h}, e(0), d\right)$ and $I_{b}(\alpha, \chi, e(0), d)$.)

The Taylor approximation of $I$ with respect to $\alpha$ shows that the bifurcation equation has two possible solutions:

$$
\begin{aligned}
& e(0) \alpha_{1}^{\prime}=\frac{9975797}{1336875}+\frac{56156 e(0)}{11625}+\frac{23}{15} e(0)^{2}+\frac{4}{3} e(0) d+\frac{21148}{10695} d+\frac{2}{10695} \sqrt{\Delta^{\prime}}, \\
& e(0) \alpha_{2}^{\prime}=\frac{9975797}{1336875}+\frac{56156}{11625} e(0)+\frac{23}{15} e(0)^{2}+\frac{4}{3} e(0) d+\frac{21148}{10695} d-\frac{2}{10695} \sqrt{\Delta^{\prime}},
\end{aligned}
$$

where $\Delta^{\prime}=-246675000 e(0) d-283676250 e(0)^{2}+164727150 e(0)-156850194-$ $145209000 d+144000000 d^{2}$. If $d$ is chosen such that $\Delta^{\prime}=0$, thus $\alpha_{1}=\alpha_{2}=\alpha^{\prime}=$ $9975797 / 1336875+(56156 / 11625) e(0)+(23 / 15) e(0)^{2}+(4 / 3) e(0) d+(21148 / 10695) d / e(0)$.

This equation admits at least one solution $d^{\prime}$ in function of $e(o)$ :

$$
d^{\prime}=\frac{3289}{3840} e(0)+\frac{48403}{96000}-\frac{1}{19200} \sqrt{\left(996649225 e(0)^{2}-103306570 e(0)+495250513\right)} .
$$

Thus there exists one and only one solution $e^{\prime}$ of the error dynamic (4.9) for any value of $e(0)$ in a neighborhood of $e=0$ given by

$$
e^{\prime}=\alpha^{\prime} e_{L}+\left(1-\alpha^{\prime}\right) e(0)+w^{*}\left(\alpha^{\prime}, \chi, e(0)\right) .
$$

Remark 4.1. Unfortunately, $e(0)$ is not known, consequently a realistic and computable correction without the knowledge of $e(0)$ consists in choosing $d$ such that the two bifurcation's solutions $\alpha_{1}^{\prime}$ and $\alpha_{2}^{\prime}$ are $\epsilon$ very closed, for all $\epsilon>0$.

Computations show that for any $e(0) \in]-1 / 5,1 / 5[$ and any choice of $d \in[0,5]$, we have that $\left|\alpha_{1}^{\prime}-\alpha_{2}^{\prime}\right|<4.1596 \times 10^{-5}$; see Figure 4.4 .

4.3. Observer design for Lagrangian system. Let us consider a Lagrangian system given by

$$
\begin{gathered}
\dot{\theta}=\omega, \\
J \dot{\omega}=\Gamma-F \omega^{2}-k \omega, \\
y=\theta,
\end{gathered}
$$




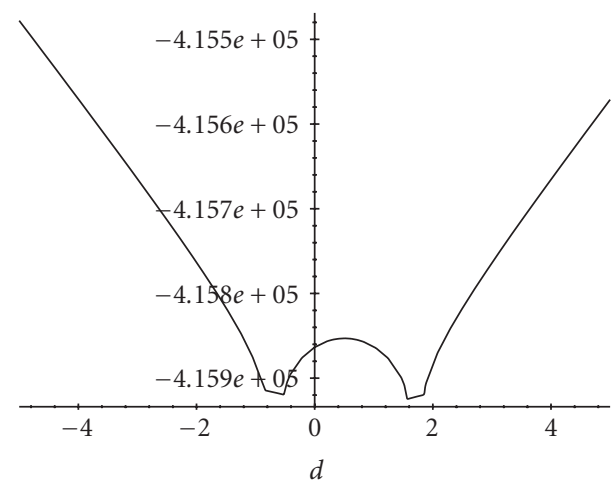

Figure 4.4

where $\theta$ is the angular position, $\omega$ is the angular velocity, $\Gamma$ is the nonnull input computed torque, $J$ is the inertia, $F$ and $k$ are disturbance parameters, and $y$ is the output of the system (4.16).

We can rewrite (4.16) as

$$
\begin{gathered}
\dot{\theta}=\omega, \\
\dot{\omega}=\Omega-\digamma \omega^{2}-K \omega, \\
y=\theta,
\end{gathered}
$$

where $\Omega=\Gamma / J, \digamma=F / J$, and $K=k / J$.

So, denoting $x_{1}=\theta$ and $x_{2}=\omega$, the system (4.17) can be rewritten as follows:

$$
\begin{gathered}
\dot{x}_{1}=x_{2}, \\
\dot{x}_{2}=\Omega-\digamma x_{2}^{2}-K x_{2} .
\end{gathered}
$$

Now, considering that the linear estimation $\hat{x}_{2}$ of $x_{2}$ is given by the following reduced observer, it comes that

$$
\begin{gathered}
\hat{x}_{2}=k y+z, \\
\dot{z}=\Omega-(k+K) \hat{x}_{2} .
\end{gathered}
$$

So, the dynamics of the observation error $e=x_{2}-\hat{x}_{2}$ satisfy the following equation:

$$
\dot{e}=-(K+k) e-\digamma\left(e+\hat{x}_{2}\right)^{2} .
$$

Here, $F=3$.

Following the notations of Section 2.4, $a(t)=-(k+K)$ is a constant gain, $\hat{x}_{2}=\chi$ is an input, and $\gamma(e, \chi, t)=-3(e+\chi)^{2}$ verifies Assumptions 2.1, 2.2, and 2.15. 
The same numerical procedure as in Section 4.1 allows to show that the approximated auxiliary equation at order one is

$$
G_{c}\left(w_{h}, \alpha, \chi_{h}, e(0)\right):=G\left(w_{h}, \alpha, \chi_{h}, e(0)\right)=0 .
$$

The resolution of this equation at order one gives

$$
a=-\frac{1}{3 \alpha}\left(-\frac{1}{25} 3-\frac{2}{5} 3 e(0)-3 e(0)^{2}\right)
$$

Replacing $a$ in the bifurcation equation, it comes that

$$
I_{c}\left(\alpha, \chi_{h}, e(0)\right):=I\left(\alpha, \chi_{h}, e(0)\right)=0 .
$$

(See Appendix D for the computation of $G_{c}\left(w_{h}, \alpha, \chi_{h}, e(0)\right)$ and $I_{c}\left(\alpha, \chi_{h}, e(0)\right)$.)

This equation has no local solution for any $e(0) \in]-1 / 5,1 / 5[$ and $e(0) \neq 0$, and so (4.20) has no solution in the neighborhood of the related linear one for any $e(0) \in$ ] $-1 / 5,1 / 5[$ and $e(0) \neq 0$.

4.4. Bifurcation correction. In order to eliminate the no solution's case, a new observer is proposed:

$$
\begin{gathered}
\hat{x}_{2}=k y+z+t d, \\
\dot{z}=\Omega-(k+K) x_{2},
\end{gathered}
$$

where $d$ is a real parameter.

The new observer error is

$$
\dot{e}=-(K+k) e-3\left(e+\hat{x}_{2}\right)^{2}+d .
$$

Then the approximated auxiliary equation is

$$
G_{d}\left(w_{h}, \alpha, \chi_{h}, e(0), d\right):=G\left(w_{h}, \alpha, \chi_{h}, e(0), d\right)=0,
$$

where $\chi_{h}=-3 t+0.2$,

$$
\begin{gathered}
\bar{e}=\alpha\left(1-\frac{3}{10} t\right) e(o)+(1-\alpha) e(0)-3 \alpha a t \\
a=-\frac{1}{3 \alpha}\left(-\frac{1}{25} 3+d-\frac{2}{5} 3 e(0)-3 e(0)^{2}\right) \text { is the solution of this equation. }
\end{gathered}
$$

Replacing $a$ in the approximated bifurcation equation, it comes that

$$
I_{d}\left(w_{h}, \alpha, \chi_{h}, e(0), d\right):=I\left(w_{h}, \alpha, \chi_{h}, e(0), d\right)=0 .
$$

(See Appendix E for the computation of $G_{d}\left(w_{h}, \alpha, \chi_{h}, e(0), d\right)$ and $I_{d}\left(w_{h}, \alpha, \chi_{h}, e(0), d\right)$.) 


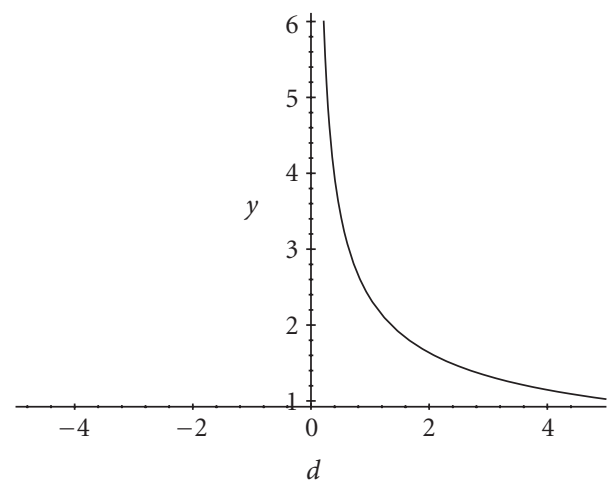

Figure 4.5

$I$ admits two solutions:

$$
\begin{aligned}
& \alpha_{1}=\frac{1}{6975 e(0)^{2}}\left(-65340 e(0)+23250 d e(0)+8100 e(0)^{2}-69750 e(0)^{3}-150 \sqrt{2} \sqrt{\Delta}\right), \\
& \alpha_{2}=\frac{1}{6975 e(0)^{2}}\left(-65340 e(0)+23250 d e(0)+8100 e(0)^{2}-69750 e(0)^{3}+150 \sqrt{2} \sqrt{\Delta}\right),
\end{aligned}
$$

where $\Delta=-429 e(0)^{2}-4290 e(0)^{3}-10725 e(0)^{4}+13175 d e(0)^{2}$ and will be equal to 0 if we choose $d=\left(1 / 13175 e(0)^{2}\right)\left(429 e(0)^{2}+4290 e(0)^{3}+10725 e(0)^{4}\right)$ and so we obtain a double solution of (4.28):

$$
\alpha_{0}=\frac{1}{6975 e(0)^{2}}\left(-65340 e(0)+23250 d e(0)+8100 e(0)^{2}-69750 e(0)^{3}\right) .
$$

This $\alpha_{0}$ corresponds to one solution of the error observation (4.25) given by

$$
e_{0}=\alpha_{0} e_{L}+\left(1-\alpha_{0}\right) e(0)+w^{*}\left(\alpha_{0}, \chi, e(0)\right) .
$$

Nevertheless, as $e(0)$ is not known and as the problem (4.16) has no solution near the linear one, an interesting correction consists in choosing $d$ such that $\left|\alpha_{1}-\alpha_{2}\right|<\varepsilon$, where $\varepsilon>0$ is chosen very small.

So for any $e(0) \in[0,1 / 5]$, and any $d>0$ it is always possible; see Figure 4.5.

\section{Conclusion}

In this paper, the analysis of the solution's bifurcation has been dealt with special attention pointed to the observation error dynamics. Contrarily to the classical ones (stability, controllability, and observability bifurcations), this type of bifurcation is not well known. Nevertheless, considering a robustness problem, solution's bifurcation is of practical importance. This fact has been highlighted in the case of linear observer design for nonlinear Lagrangian systems. This type of bifurcation is also of the great interest in the study of population dynamics, specially when a parameter changes and causes an explosion of demography due to a chain reaction. 


\section{Appendices}

\section{A. Design of the correction term}

Auxiliary equation analysis (3.11a). The correction term is

$$
\tilde{v}=(1-\alpha) e(0)+\widetilde{w}, \tilde{w} \in\left(\mathrm{Id}-P_{0}\right) C^{1}
$$

and the operator $\tilde{G}:\left(\operatorname{Id}-P_{0}\right) C^{1} \times \mathbb{R} \times X \times \mathbb{R}^{2} \rightarrow\left(\mathrm{Id}-P_{0}\right) C^{1}$ is defined as

$$
\widetilde{G}(\widetilde{w}, \alpha, \chi, e(0), d)=(1-\alpha) e(0)+\widetilde{w}-K P_{1} \hat{N}\left(\alpha e_{L}+(1-\alpha) e(0)+\widetilde{w}, \chi, d\right)
$$

The first step is to study the number of solutions $\widetilde{w}$ of the equation: $\widetilde{G}(w, \alpha, \chi, e(0)$, d) $=0$.

From Assumptions 2.1 and 2.2, it can be deduced that $\widetilde{G}$ verifies the following conditions:

(i) $\widetilde{G}(0,0,0,0,0)=0$;

(ii) $\widetilde{G}$ is $C^{2}$ with respect to $\widetilde{w}$;

(iii) $D_{\widetilde{w}} \widetilde{G}(0,0,0,0,0)=\left.\operatorname{Id}\right|_{\left(\mathrm{Id}-P_{0}\right) C^{1}}$.

Now, using the implicit function theorem, it comes to the following.

Corollary A.1. There exist a neighborhood $V_{\widetilde{w}}$ of $\widetilde{w}=0$ in $\left(\mathrm{Id}-P_{0}\right) C^{1}$, a neighborhood $V_{\alpha}$ of $\alpha=0$ in $\mathbb{R}$, a neighborhood $V_{\chi}$ of $\chi=0$ in $X$, a neighborhood $V_{e(0)}$ of $e(0)=0$ in $\mathbb{R}$, and a neighborhood $V_{d}$ of $d=0$ in $\mathbb{R}$ such that for $(\alpha, \chi, e(0), d) \in V_{\alpha} \times V_{\chi} \times V_{e(0)} \times V_{d}$, the auxiliary equation (3.11a) admits one and only one solution $\widetilde{w}^{*}(\alpha, \chi, e(0), d) \in V_{\widetilde{w}}$ such that $\widehat{w}^{*}(0,0,0,0)=0$ if and only if $(\alpha, \chi, e(0), d)$ is a solution of bifurcation equation (3.11a).

Bifurcation equation analysis. The system (3.11) admits a solution if and only if $\alpha$ satisfies the bifurcation equation (3.11b). This becomes

$$
\tilde{I}(\alpha, \chi, e(0), d)=\int_{0}^{T} \tilde{\gamma}\left(\alpha e_{L}+(1-\alpha) e(0)+\tilde{w}^{*}(\alpha, \chi, e(0), d), \chi, d, t\right) e_{L}(t) d t=0 .
$$

Case 1. If there exist $\alpha_{0}, \chi_{0}, e(0)$, and $d_{0}$ such that

$$
\begin{gathered}
\tilde{I}\left(\alpha_{0}, \chi_{0}, e(0), d_{0}\right)=0, \\
\frac{\partial \tilde{I}}{\partial \alpha}\left(\alpha_{0}, \chi_{0}, e(0), d_{0}\right) \neq 0 .
\end{gathered}
$$

In this case, the implicit function theorem ensures that there exist a neighborhood $V_{\alpha_{0}}$ of $\alpha_{0}$ in $\mathbb{R}$, a neighborhood $\tilde{\mathscr{V}}_{\chi_{0}}$ of $\chi_{0}$ in $X$, a neighborhood $\tilde{\mathscr{V}}_{e_{f}(0)}$ of $e(0)$ in $\mathbb{R}$, a neighborhood $V_{d_{0}}$ of $d_{0}$, and one and only one solution $\tilde{\alpha}^{*}: V_{\chi_{0}} \times V_{e_{f}(0)} \times V_{d_{0}} \rightarrow V_{\alpha_{0}}$ such that $\tilde{\alpha}^{*}\left(\chi_{0}, e(0), d_{0}\right)=\alpha_{0}$ and $\tilde{I}\left(\tilde{\alpha}^{*}\left(\chi, e(0), d_{0}\right), \chi, e(0), d_{0}\right)=0$, for all $e(0) \in V_{e(0)}$, for all $\chi \in \tilde{\mathscr{V}}_{\chi_{0}}$, for all $d \in \tilde{\mathscr{V}}_{d_{0}}$. 
In this case, the problem (3.10) admits one solution $\tilde{e}^{*}=\tilde{\alpha}^{*}(\chi, e(0), d) e_{L}+\left(1-\tilde{\alpha}^{*}(\chi\right.$, $e(0), d) e(0))+w^{*}\left(\tilde{\alpha}^{*}(\chi, e(0), d), \chi, e(0)\right)$ near $e_{L}$ and there is no bifurcation.

Case 2. $\exists v_{\alpha} \times v_{\chi} \times v_{e(0)} \times v_{d} \subset V_{\alpha} \times V_{\chi} \times V_{e(0)} \times V_{d}$ such that, for all $(\alpha, \chi, e(0), d) \in v_{\alpha} \times$ $v_{\chi} \times v_{e(0)} \times v_{d}:$

$$
\widetilde{I}(\alpha, \chi, e(0), d)=0 \Longrightarrow \frac{\partial \tilde{I}}{\partial \alpha}(\alpha, \chi, e(0), d)=0
$$

from Assumption 2.15, it comes

$$
\frac{\partial^{2} \tilde{I}}{\partial \alpha^{2}}(0,0,0,0) \neq 0
$$

and the implicit functions theorem applied to $(\partial \tilde{I} / \partial \alpha)(\alpha, \chi, e(0), d)=0$ ensures that there exist a neighborhood $\bar{v}_{\alpha} \subset v_{\alpha}$ of $\alpha=0$ in $\mathbb{R}$, a neighborhood $\bar{v}_{\chi} \subset v_{\chi}$ of $\chi=0$ in $X$, a neighborhood $\bar{v}_{e(0)} \subset v_{e(0)}$ of $e(0)=0$ in $\mathbb{R}$, a neighborhood $\bar{v}_{d} \subset v_{d}$ of $d=0$ in $\mathbb{R}$, and a unique application $\tilde{\alpha}^{* *}: \bar{v}_{\chi} \times \bar{v}_{e(0)} \times \bar{v}_{d} \rightarrow \bar{v}_{\alpha}$ such that $\alpha^{* *}(0,0,0)=0$ and $(\partial \tilde{I} / \partial \alpha)\left(\tilde{\alpha}^{* *}(\chi, e(0))\right.$, $\chi, e(0), d)=0$, for all $\chi \in \bar{v}_{\chi}$, for all $e(0) \in \bar{v}_{e(0)}$, for all $d \in \bar{v}_{d}$. Therefore, we can write the Taylor expansion at order 2 near $\alpha^{* *}$ of $\tilde{I}$

$$
\begin{aligned}
\widetilde{I}(\alpha, \chi, e(0), d)= & \widetilde{I}\left(\tilde{\alpha}^{* *}(\chi, e(0), d), \chi, e(0)\right) \\
& +\frac{1}{2} \frac{\partial^{2} \tilde{I}}{\partial \alpha^{2}}\left(\tilde{\alpha}^{* *}(\chi, e(0), d), \chi, e(0), d\right)\left(\alpha-\tilde{\alpha}^{* *}(\chi, e(0), d)\right)^{2} \\
& +O\left(\left|\alpha-\tilde{\alpha}^{* *}(\chi, e(0))\right|^{2}\right)
\end{aligned}
$$

when $\alpha \rightarrow \tilde{\alpha}^{* *}$ and we have to solve

$$
\tilde{I}(\alpha, \chi, e(0), d)=O\left(\left|\alpha-\tilde{\alpha}^{* *}\right|^{3}\right)
$$

and as $\tilde{v}_{\widetilde{\alpha}^{* *}}$ is sufficiently small $\left(\partial^{2} \tilde{I} / \partial \alpha^{2}\right)\left(\tilde{\alpha}^{* *}(\chi, e(0)), \chi, e(0), d\right)$ has the same sign as $\left(\partial^{2} \tilde{I} / \partial \alpha^{2}\right)(0, \chi, e(0), d)$, so the following possibilities appear:

(i) when $\left(\partial^{2} \tilde{I} / \partial \alpha^{2}\right)(0, \chi, e(0), d)>0$ and $I\left(\tilde{\alpha}^{* *}, \chi, e(0)\right)<0$ then (A.8) has two solutions in the neighborhood of $\alpha^{* *}$,

(ii) when $\left(\partial^{2} \tilde{I} / \partial \alpha^{2}\right)(0, \chi, e(0), d)<0$ and $I\left(\tilde{\alpha}^{* *}, \chi, e(0)\right)>0$ then (A.8) has two solutions in the neighborhood of $\tilde{\alpha}^{* *}(\chi, e(0), d)$,

(iii) when $\left(\partial^{2} \tilde{I} / \partial \alpha^{2}\right)(0, \chi, e(0), d)>0$ and $I\left(\tilde{\alpha}^{* *}, \chi, e(0)\right)>0$ then (A.8) has no solution in the neighborhood of $\tilde{\alpha}^{* *}(\chi, e(0), d)$,

(iv) when $\left(\partial^{2} \hat{I} / \partial \alpha^{2}\right)(0, \chi, e(0), d)<0$ and $I\left(\tilde{\alpha}^{* *}, \chi, e(0)\right)<0$ then $($ A.8) has no solution in the neighborhood of $\tilde{\alpha}^{* *}(\chi, e(0), d)$,

(v) when $I\left(\tilde{\alpha}^{* *}, \chi, e(0)\right)=0$, then $\left(\right.$ A.8) has one solution $\tilde{\alpha}^{* *}$. 


\section{B. Approximated bifurcation equation without correction for (4.1)}

$$
\begin{aligned}
G_{a}\left(\alpha, \chi_{h}, e(0)\right) & \\
= & -3 \alpha a t+\left(1-\frac{3}{10} t\right) \int_{0}^{t}\left(1+\frac{3}{10} s\right) 2(-3 s+0.2) \\
& \times\left\{\alpha\left(1-\frac{3}{10} s\right) e(0)+(1-\alpha) e(0)+\alpha\left(\frac{1}{25} e(0) \frac{2(0.2)-e(0)}{\alpha(-3)}\right)(-3) s\right. \\
& \left.-\left(\alpha\left(1-\frac{3}{10} s\right) e(0)+(1-\alpha) e(0)+\alpha\left(\frac{1}{25} e(0) \frac{2(0.2)-e(0)}{\alpha(-3)}\right)(-3) s\right)^{2}\right\} d s \\
I_{a}\left(\alpha, \chi_{h}, e(0)\right) & \int_{0}^{1}\left(1-\frac{3}{10} s\right) 2(-3 s+0.2)\left\{\alpha\left(1-\frac{3}{10} s\right) e(0)+(1-\alpha) e(0)\right. \\
& +\alpha\left(\frac{1}{25} e(0) \frac{2(0.2)-e(0)}{\alpha(-3)}\right)(-3) s-\left(\alpha\left(1-\frac{3}{10} s\right) e(0)+(1-\alpha) e(0)\right. \\
& \left.\left.+\alpha\left(\frac{1}{25} e(0) \frac{2(0.2)-e(0)}{\alpha(-3)}\right)(-3) s\right)^{2}\right\} d s .
\end{aligned}
$$

\section{Approximated bifurcation equation with correction for (4.1)}

$$
\left.\begin{array}{l}
G_{b}\left(w_{h}, \alpha, \chi_{h}, e(0), d\right) \\
=-3 \alpha a t-\left(1-\frac{3}{10} t\right) \int_{0}^{t}\left(1+\frac{3}{10} s\right) \\
\times\left(\begin{array}{c}
\frac{6}{100}\left((\alpha e(0)(1-0,3 s)+(1-\alpha) e(0)-3 \alpha a s)^{2}\right. \\
-2\left(\alpha e(0)\left(1-\frac{3}{10} s\right)+(1-\alpha) e(0)-3 \alpha a s\right) \\
\left.\left(\frac{2}{10}-3 s\right)\right)+\frac{1}{10}\left(\frac{2}{10}-3 s\right)^{2}-\frac{4}{10}\left(\frac{2}{10}-3 s\right. \\
\left.-\left(\alpha e(0)\left(1-\frac{3}{10} s\right)+(1-\alpha) e(0)-3 \alpha a s\right)\right) \\
\left(\left(\alpha e(0)\left(1-\frac{3}{10} s\right)+(1-\alpha) e(0)-3 \alpha a s\right)+d+\frac{3}{10}\left(\frac{2}{10}-3 s\right)^{2}\right.
\end{array}\right)
\end{array}\right)
$$


26 Lyapunov-Schmidt method and observer analysis

$$
\begin{aligned}
& I_{b}(\alpha, \chi, e(0), d) \\
& =\int_{0}^{1}\left(1-\frac{3}{10} s\right) \\
& \times\left(\frac { 6 } { 1 0 0 } \left\{\left(\begin{array}{c}
\alpha e(0)\left(1-\frac{3}{10} s\right)+(1-\alpha) e(0) \\
-3 \alpha\left(-\frac{1}{18750} \frac{2875 e(0)^{2}+19-620 e(0)-500 d+2500 e(0) d}{\alpha}\right) s
\end{array}\right)^{2}\right.\right. \\
& \times\left(\begin{array}{c}
\alpha e(0)\left(1-\frac{3}{10} s\right)+(1-\alpha) e(0) \\
-3 \alpha\left(-\frac{1}{18750} \frac{2875 e(0)^{2}+19-620 e(0)-500 d+2500 e(0) d}{\alpha}\right) s
\end{array}\right) \\
& \left.\times\left(\frac{2}{10}-3 s\right)\right\}+\frac{1}{10}\left(\frac{2}{10}-3 s\right)^{2}-\frac{4}{10}\left(\frac{2}{10}-3 s\right. \\
& -\left(\begin{array}{c}
\alpha e(0)\left(1-\frac{3}{10} s\right)+(1-\alpha) e(0) \\
-3 \alpha\left(-\frac{1}{18750} \frac{2875 e(0)^{2}+19-620 e(0)-500 d+2500 e(0) d}{\alpha}\right) s
\end{array}\right) \\
& \times\left(\left(\alpha e(0)\left(1-\frac{3}{10} s\right)+(1-\alpha) e(0)\right.\right. \\
& \left.-3 \alpha\left(-\frac{1}{18750} \frac{2875 e(0)^{2}+19-620 e(0)-500 d+2500 e(0) d}{\alpha}\right) s\right) \\
& \left.+d+\frac{3}{10}\left(\frac{2}{10}-3 s\right)^{2}\right) d s .
\end{aligned}
$$

\section{Approximated bifurcation equation without correction for (4.16)}

$$
\begin{aligned}
G_{c}\left(w_{h}, \alpha, \chi_{h}, e(0)\right)= & -3 \alpha a t-\left(1-\frac{3}{10} t\right) \int_{0}^{t}\left(1+\frac{3}{10} s\right) \\
& \times\left(-3\left(\left(\begin{array}{c}
\alpha\left(1-\frac{3}{10} s\right) e(0) \\
+(1-\alpha) e(0)-3 \alpha a s
\end{array}\right)\right)+\left(-3 s+\frac{2}{10}\right)\right)^{2} d s,
\end{aligned}
$$




$$
\begin{aligned}
& I_{c}\left(\alpha, \chi_{h}, e(0)\right)=\int_{0}^{1}\left(1+\frac{3}{10} s\right)\left(-3\left(\begin{array}{c}
\alpha\left(1-\frac{3}{10} s\right) e(0)+(1-\alpha) e(0) \\
-3 \alpha\left(-\frac{1}{3 \alpha}\left(-\frac{1}{25} 3-\frac{2}{5} 3 e(0)-3 e(0)^{2}\right)\right) s
\end{array}\right)\right) \\
& \left.+\left(-3 s+\frac{2}{10}\right)\right)^{2} d s
\end{aligned}
$$

\section{E. Approximated bifurcation equation with correction for (4.16)}

$$
\begin{gathered}
G\left(w_{h}, \alpha, \chi_{h}, e(0), d\right)=-3 \alpha a t-\left(1-\frac{3}{10} t\right) \int_{0}^{t}\left(1+\frac{3}{10} s\right)\left(-3(\bar{e}+\chi)^{2}+d\right) d s \\
I\left(w_{h}, \alpha, \chi_{h}, e(0), d\right)=\int_{0}^{1}\left(1-\frac{3}{10} s\right)\left(-3\left(\begin{array}{c}
\alpha\left(1-\frac{3}{10} s\right) e(0)+(1-\alpha) e(0) \\
\left.\left.-3 \alpha\left(-\frac{1}{3 \alpha}\left(-\frac{1}{25} 3+d-\frac{2}{5} 3 e(0)-3 e(0)^{2}\right)\right) s\right)\right) \\
+
\end{array}\right.\right. \\
\left.+\left(-3 s+\frac{2}{10}\right)^{2}+d\right) d s .
\end{gathered}
$$

\section{Acknowledgments}

The authors are very grateful to Professor H. Mokhtar-Kharoubi from Oran University (Algeria) for his suggestions and helpful remarks. These have largely contributed to the improvement of this paper.

\section{References}

[1] E. H. Abed, H. O. Wang, and A. Tesi, Control of bifurcation and Chaos, The Control Handbook (W. S. Levine, ed.), CRC Press, Florida; IEEE Press, New Jersey, 1995.

[2] V. I. Arnold, V. S. Afraimovitch, Yu. Ilyashenko, and L. P. Shilnikov, Theory of Bifurcations, Encyclopedia of Mathematics, vol. 5, Springer, New York, 1982.

[3] D. Benmerzouk and J. P. Barbot, Observability analysis using Lyapunov-Schmidt method, Proceedings of Symposium on Nonlinear Control Systems, vol. 1, Stuttgart, 2004, pp. 323-328.

[4] L. Boutat-Baddas, D. Boutat, J. P. Barbot, and R. Tauleigne, Quadratic observability normal form, part I, Proceedings of 40th IEEE Conference on Decision and Control, Florida, 2001, pp. 29422947.

[5] M. Demazure, Bifurcation and Catastrophes, Geometry of Solution of Nonlinear Problem, Springer, New York, 1991.

[6] W. Kang, Bifurcation and normal form of nonlinear control systems. I, SIAM Journal on Control and Optimization 36 (1998), no. 1, 193-212. 
[7] __ Bifurcation and normal form of nonlinear control systems. II, SIAM Journal on Control and Optimization 36 (1998), no. 1, 213-232.

[8] W. Kang and A. J. Krener, Extended quadratic controller normal form and dynamic state feedback linearization of nonlinear systems, SIAM Journal on Control and Optimization 30 (1992), no. 6, 1319-1337.

[9] H. K. Khalil, Nonlinear Systems, 2nd ed., Prentice-Hall, New Jersey, 1996.

[10] J. E. Marsden, Qualitative methods in bifurcation theory, Bulletin of the American Mathematical Society 84 (1978), no. 6, 1125-1148.

[11] J.-C. Paumier, Bifurcations et Méthodes Numériques, Recherches en Mathématiques Appliquées, Masson, Paris, 1997.

[12] S. Wiggins, Introduction to Applied Nonlinear Dynamical Systems and Chaos, Texts in Applied Mathematics, vol. 2, Springer, New York, 1990.

D. Benmerzouk: Département de Mathématique et d'Informatique, Institut de Mathèmatique, Université Abou Bekr Belkaid Tlemcen, BP 119, 13000 Tlemcen, Algeria

E-mail address: d_benmerzouk@univ_tlemcen.dz

J. P. Barbot: Equipe Commande des Systèmes (ECS)-EA 3649, ENSEA, 6 Avenue du Ponceau, 95014 Cergy-Pontoise Paris, France

E-mail address: barbot@ensea.fr 


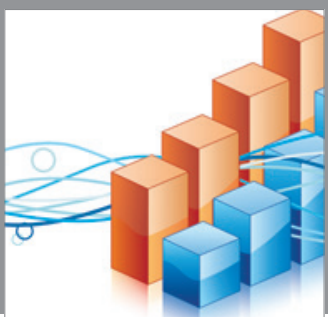

Advances in

Operations Research

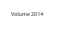

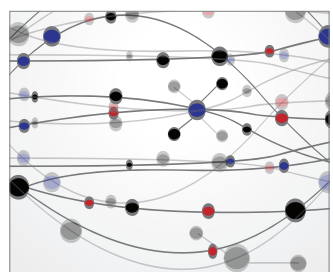

\section{The Scientific} World Journal
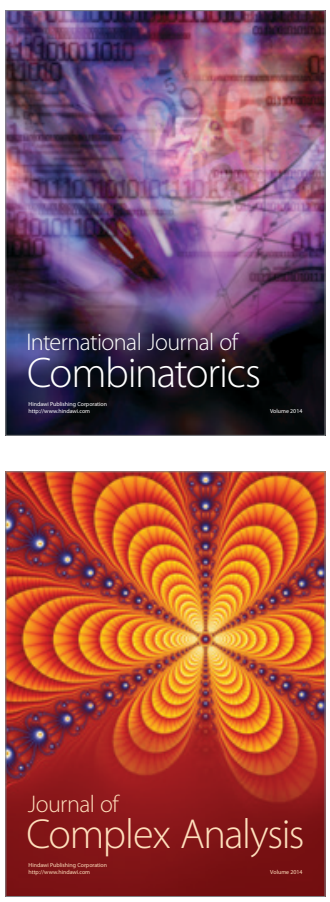

International Journal of

Mathematics and

Mathematical

Sciences
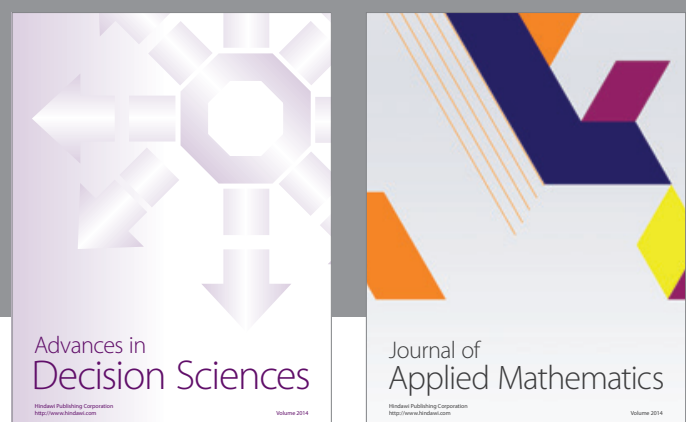

Journal of

Applied Mathematics
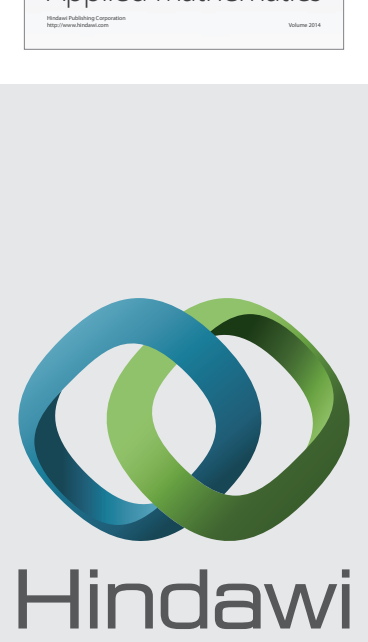

Submit your manuscripts at http://www.hindawi.com
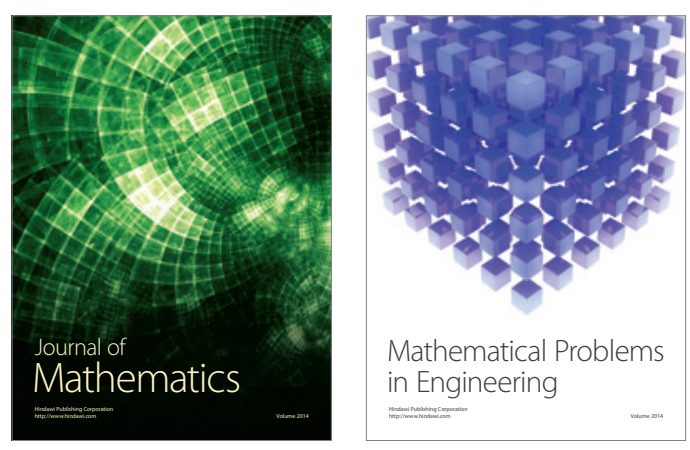

Mathematical Problems in Engineering
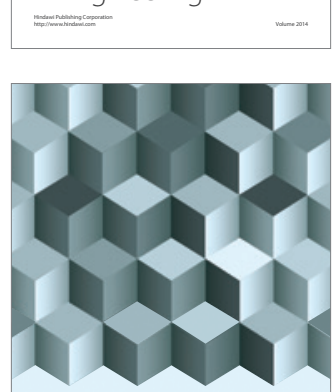

Journal of

Function Spaces
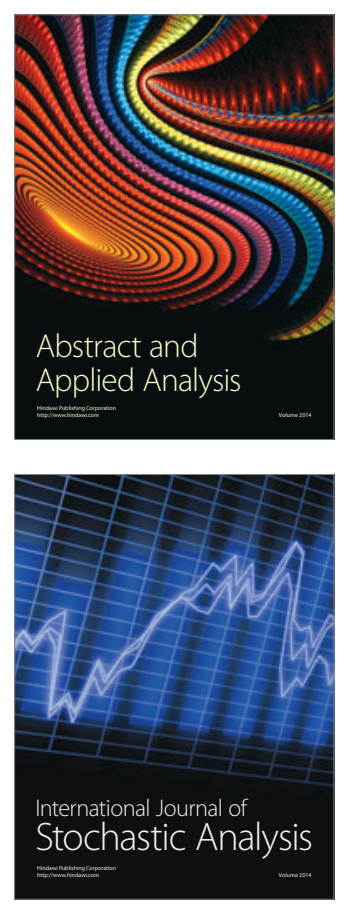

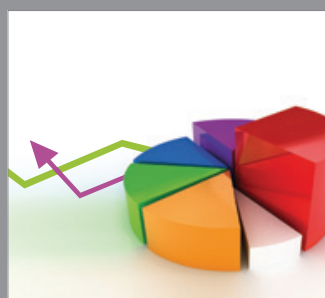

ournal of

Probability and Statistics

Promensencen
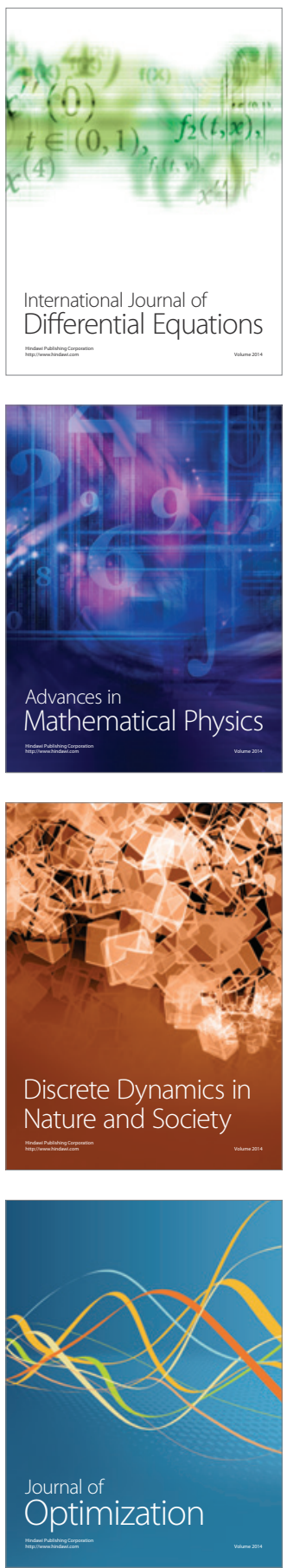